\title{
A stress-based approach to the optimal design of structures with unilateral behavior of material or supports
}

\author{
Matteo Bruggi • Pierre Duysinx
}

Received: date / Accepted: date

\begin{abstract}
The paper presents a stress-based approach that copes with the optimal design of truss-like elastic structures in case of unilateral behavior of material or ground supports. The conventional volume-constrained minimization of compliance is coupled with a set of local stress constraints that are enforced, all over the domain or along prescribed boundaries, to control the arising of members with tension-only or compression-only strength. A Drucker-Prager failure criterion is formulated to provide a smooth approximation of the no-tension or no-compression conditions governing the stress field. A selection strategy is implemented to handle efficiently the multi-constrained formulation that is solved through mathematical programming. Benchmark examples are investigated to discuss the features of the achieved optimal designs, as compared with problems involving material and ground supports with equal behavior in tension and compression. Numerical simulations show that a limited set of constraints is needed in the first iterations to steer the solution of the energy-driven optimization towards designs accounting for the prescribed assumption of unilateral strength.
\end{abstract}

Matteo Bruggi

Department of Civil and Environmental Engineering

Politecnico di Milano, I20133, Milano, Italy

Tel.: +39-02-23994232

Fax: +39-02-23994220

E-mail: matteo.bruggi@polimi.it

Pierre Duysinx

Department of Aerospace and Mechanical Engineering

University of Liège, 4000 Liège, Belgium

Tel.: +32-4-3669194

Fax: +32-4-3669159

E-mail: p.duysinx@ulg.ac.be
Keywords topology optimization - stress constraints unilateral materials · unilateral supports · DruckerPrager strength criterion

\section{Introduction}

Since the pioneering investigations of Bendsøe and Kikuchi (1988), topology optimization has been widely concerned with the classic approach of minimum compliance subject to a volume constraint. Strain energy is the objective function to be minimized with the aim of achieving stiff truss-like structures that employ a limited amount of material. The stiffness-based method has been extended to several needs of structural engineering such as maximizing the fundamental natural frequency, increasing the ultimate loads for material strength or designing compliant mechanisms, among others. Reference is made to e.g. Eschenauer and Olhoff (2001), Bendsøe and Sigmund (2003) and Rozvany (2009) for comprehensive literature reviews.

Most of the available formulations are conceived to cope with elastic media exhibiting the same behavior in tension and compression and cannot be straightforwardly adopted to perform any conceptual design of a wide class of structures involving building materials with tension-only or compression-only strength. To close this gap, alternative methods have been proposed in the literature of the last decades. Chang et al. (2007) implemented the unequal behavior in tension and compression through the adoption of a non-linear constitutive law for the material to be optimized, see e.g. Medri (1982). This direct approach provides a robust modeling of the structural behavior but remarkably increases the cost of the finite elements analysis with respect to conventional linear problems, since iterative solvers are 
needed to compute an accurate displacement field before each update of the design variables.

Alternatively, many efforts were directed in the literature towards the development of numerical strategies accounting for the non-symmetric behavior of the material without coping with an expensive direct implementation of non-linear constitutive equations. Guan et al. (1999) tackled the problem resorting to the optimization of the so-called material-oriented structures and adopted the ESO method (Xie and Steven 1993) to distribute phases resisting the tensile or compressive forces depending on the principal stresses on the domain. Remodeling theories were also used to provide optimal design where unilateral material is oriented depending on the stress flows directions or the strain energy density, see e.g. Pàlfi (2004) and Novak (2006). Querin et al. (2010) proposed to cope with nonsymmetric materials through a conversion of the original isotropic properties to orthotropic ones, iteratively modifying the modulus of elasticity and stresses/strains of the elements while satisfying the optimality criterion of a constant stress/strain ratio in the structure, see Dewhurst (2005). Dealing with no-tension and nocompression structures, Cai (2011) suggested an efficient material-replacement strategy that performs the substitution of a conventional symmetric phase with a new isotropic material, providing the desired unilateral properties within the optimization algorithm. Reference is also made to Lie and Qiao (2011) that considered different stiffness of concrete and steel in order to generate optimal layouts with distinct tension and compression members to address conceptual design of bridges.

Remaining in the field of structural topology optimization, it is worth remarking that the assumption of unilateral behavior does not refer only to materials but may also apply to boundary constraints. In many applications of civil engineering one has to cope with non bilateral supports that provide a compressive reaction while being inactive in tension. Common examples are ground constraints for buildings or certain connections among structural components.

This problem has been generally regarded in the literature as an application of challenging formulation for topology optimization involving contact mechanics. Several methods have been proposed in the literature to address the Signorini's conditions within different numerical approaches. Petersson and Patriksson (1997) presented a pioneering work on the topology optimization of sheets in unilateral contact resorting to a sub-gradient method. A contact problem including Coulomb friction was tackled in Fancello (2006) through a penalization approach, while Mankame and Ananthasuresh (2004) extended investigations to the optimal design of compliant mechanisms introducing a regularized contact model. The very recent work in Strömberg and Klarbring (2010) addresses contact problems in the three-dimensional framework treating the Signorini's conditions by the augmented Lagrangian approach along with a smooth approximation technique.

Within the above framework, this contribution introduces a simple approach to the optimal design of truss-like structures under the assumption of unilateral behavior of material or ground supports. The proposed method is based on the adoption of a local control of the feasibility of the stress field, while no alternative modeling or ad hoc numerical treatment is required to cope with the evolving structure or any of its parts. A suitable form of the Drucker-Prager failure criterion is introduced to provide a smooth approximation of the sign conditions on the first and third invariant of the stress tensor that govern the assumption of unilateral strength. A set of local stress constraints is embedded in the classical energy-based formulation for isotropic materials, thus controlling the arising of no-tension or nocompression members within the bulk of the domain or in prescribed regions located along the boundaries. The multi-constrained formulation is solved through mathematical programming adopting the Method of Moving Asymptotes (Svanberg 1987) and resorting to a selection strategy previously tested in Bruggi and Duysinx (2012). Numerical simulations show that a limited number of enforcements is able to steer the solution towards truss-like layouts that fulfill the prescribed unilateral assumption concerning material or supports. In general, most of the optimization is performed as a pure volumeconstrained strain energy minimization that refines the optimal design sketched by the constraints acting in the very first iterations.

The remainder of the paper is as follows. Section 2 reports fundamentals of the considered topology optimization problem and discusses the main idea of prescribing unilateral behavior of materials or supports through a unified stress-based framework. Section 3 introduces the discrete form of the adopted multi-constrained minimum compliance setting and addresses related numerical issues such as the implementation of a selection strategy for stress constraints and the adoption of a relaxation procedure against instabilities. Section 4 presents numerical simulations to assess the approach. The achieved results are compared with conventional solutions for symmetric materials and bilateral constraints, paying attention to the features of the optimal layouts and to the convergence properties of the method. Section 5 concludes the paper and formulates remarks on the presented investigations. 


\section{Governing equations}

As introduced in Section 1, the proposed formulation copes with the design of truss-like structures in case of unilateral material or constraints enforcing the nonsymmetric behavior through a stress-based optimization. Such an approach moves from the governing equations that are found in conventional problems for minimum compliance, whose applicability to the considered framework will be discussed in the sequel.

A body made of linear elastic isotropic material is set in a two-dimensional domain $\Omega$ where no volume force is supposed to act. An orthogonal reference frame $O x_{1} x_{2}$ is adopted. The boundary of the domain $\Gamma=$ $\Gamma_{t} \cup \Gamma_{u}$ consists of two different parts, subject to traction $\underline{t}_{0}\left(\Gamma_{t}\right)$ or to prescribed displacements $\underline{u}_{0}\left(\Gamma_{u}\right)$.

The distribution of material is tackled through the material density function $0 \leq \rho(\chi) \leq 1$ that is defined in each point of the domain $\chi \in \Omega$. Following the well-known SIMP model, see e.g. Bendsøe and Kikuchi (1988) and Zhou and Rozvany (1991), one may introduce a suitable form of the fourth order elasticity tensor $C_{i j h k}(\rho(\chi))$ depending on the local value of the density function $\rho$, i.e.:

$C_{i j h k}(\rho(\chi))=\rho(\chi)^{p} C_{i j h k}^{0}$,

where $C_{i j h k}^{0}$ is the stiffness tensor for a given isotropic medium, while $p>1$ is a penalization parameter that will be assumed to be equal to 3, see e.g. Bendsøe and Sigmund (1999).

A weak formulation for the solution of the considered elastic problem may be derived in terms of displacements by means of the primal variational principle. Recalling that the relevant functional is defined as the sum of the strain energy stored in the elastic medium with constitutive tensor $C_{i j h k}(\rho(\chi))$ and the potential energy of the applied loads, one may straightforwardly write the stationarity condition for the total potential energy. It reads: find $\underline{u} \in H^{1}$ such that $\left.\underline{u}\right|_{\Gamma_{u}}=\underline{u}_{0}$ and

$\int_{\Omega} \rho^{p} C_{i j k l}^{0} \varepsilon_{i j}(\underline{u}) \varepsilon_{k l}(\underline{v}) d \Omega=\int_{\Gamma_{t}} \underline{t}_{0} \cdot \underline{v} d \Gamma$,

$\forall \underline{v} \in H^{1}$, where the strain tensor is defined as $\varepsilon_{i j}=$ $\frac{1}{2}\left(u_{i, j}+u_{j, i}\right)$ and the stress field reads:

$\sigma_{i j}(\underline{u}, \rho)=\rho^{p} C_{i j k l}^{0} \varepsilon_{k l}(\underline{u})=\rho^{p} \bar{\sigma}_{i j}(\underline{u})$.

To formulate a problem of topology optimization for maximum stiffness design, the so-called structural compliance is introduced:

$\mathcal{C}=\int_{\Omega} \rho^{p} C_{i j k l}^{0} \varepsilon_{i j}(\underline{u}) \varepsilon_{k l}(\underline{u}) d \Omega$,

that corresponds to the work of the external loads at equilibrium. Indeed, Eqn. (4) is the bilinear form at the l.h.s. of Eqn. (2) evaluated for $\underline{v}=\underline{u}$.

\subsection{Unilateral materials}

Let consider the case of a unilateral material. The notension hypothesis requires the stress tensor $\sigma_{i j}$ belong to the closed cone of negative semi-definite symmetric tensors, that is equivalent to restricting the principal stresses to be non-positive, see e.g. Medri (1982). Within the two-dimensional framework, the following two inequalities hold in $\Omega$ :

$\sigma_{i i} \leq 0, \quad \sigma_{i i} \sigma_{j j}-\sigma_{i j} \sigma_{i j} \leq 0$,

where $\sigma_{i i}$ is the trace of the stress tensor, while $\sigma_{i i} \sigma_{j j}-$ $\sigma_{i j} \sigma_{i j}$ is twice the determinant.

Recalling Eqn. (3), the set of stress constraints to be embedded within a formulation that aims at enforcing the distribution of no-tension material in $\Omega$ may be written as:

$\rho^{p} \bar{\sigma}_{i i} \leq 0, \quad \rho^{2 p}\left(\bar{\sigma}_{i i} \bar{\sigma}_{j j}-\bar{\sigma}_{i j} \bar{\sigma}_{i j}\right) \leq 0$.

Eqns. (6) prescribe the unilateral behavior for $\rho>0$, meaning that tensile material with non-zero density is not allowed. This also means that the bilinear form in Eqn. (2) and the compliance in Eqn. (4) have non-zero contributions that only refer to material stressed by a no-tension regime. Alternatively, one may re-formulate the dual problem of no-compression material by simply requiring the stress tensor $\sigma_{i j}$ belong to the closed cone of positive semi-definite symmetric tensors, i.e. changing the sign of the inequalities in Eqns. (5) and (6).

It must be remarked that the proposed approach is especially conceived to find optimal truss-like designs of minimum compliance, whose mechanical behavior is mainly governed by the uniaxial stress state acting in each strut or tie. The constitutive behavior of such kind of structures is effectively modeled in the two-dimensional domain $\Omega$ by the bilinear form of Eqn. (2) due to its dependence on $\rho$ through the constraints of Eqns. (6).

Let focus on a slender compressive member being part of a truss-like design. According to St. Venant's theory, an axially loaded strut is compressed along the main direction while a positive Poisson's ratio calls for an extension of its section. The considered isotropic material model, i.e. Eqn. (1), provides the void zones with negligible stiffness, meaning that the enlargement occurring in the full-material slender element is allowed. No transversal tension arises in the strut and it fully complies with Eqns. (6). This holds for straight bars but also for curved slender elements, such as arch-like structural components.

Focusing on a deep compressive member, it should be taken into account that the Poisson's effect may be responsible for some tension if the massive element is modeled through the isotropic penalization of Eqn. 

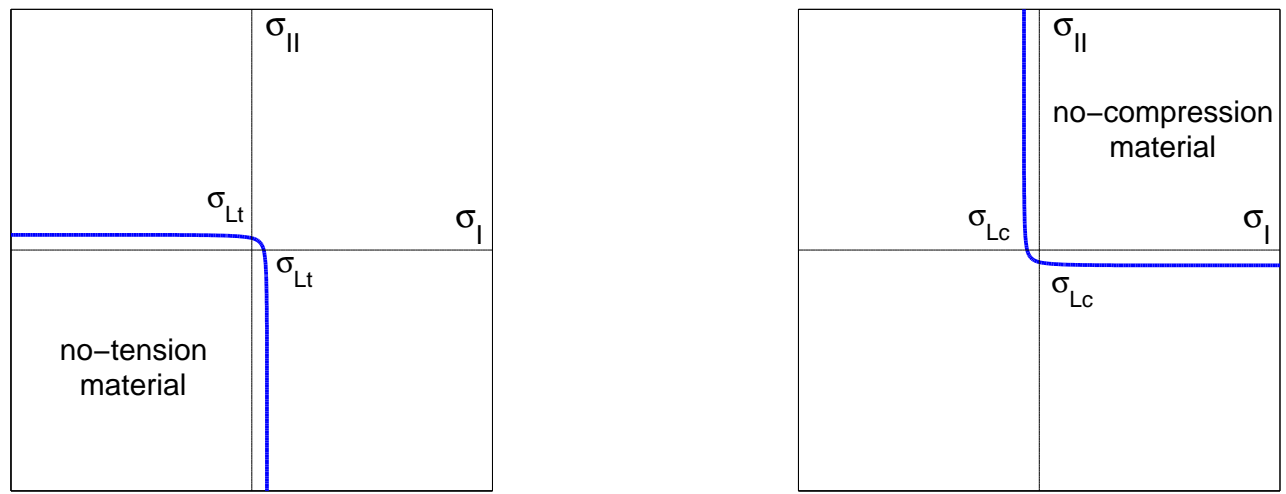

Fig. 1 Feasible domains defined by the Drucker-Prager strength function of Eqn. (7) in the plane of the principal stresses $\sigma_{I}$ and $\sigma_{I I}$ : a prescribed small value of the tensile strength $\sigma_{L t}$ along with $s=\sigma_{L c} / \sigma_{L t}=100$ handles no-tension materials (a); a prescribed small value of the compressive strength $\sigma_{L c}$ along with $s=\sigma_{L c} / \sigma_{L t}=1 / 100$ handles no-compression materials (b).

(1). The particular geometry of the structural component or its boundary conditions may introduce a nonhomogeneous limitation to the transversal strain that occurs in the bulk of the element. As a consequence, a minor principal stress arises in certain regions along with the expected major compressive one, see e.g. the test for determining the splitting tensile strength of cylindrical concrete specimens that are compressed along a diameter. This means that, in some cases, the notension requirement enforced by Eqns. (6) can not be strictly satisfied within a deep element that is tackled by the bilinear form of Eqn. (2).

The above discussion suggests that the adopted isotropic framework may be robustly applied to generate optimal truss-like designs, i.e. working with a low volume fraction of material, while optimal massive structures that are acted upon by a bi-axial stress state should be investigated resorting to more advanced anisotropic material models, see Section 1. To this purpose reference is also made to Del Piero (1989) who discusses a theoretical insight on hyperelasticity of unilateral materials and provides a rigorous derivation of a strain energy density function for no-tension materials under plane stress or plane strain conditions.

\subsection{A smooth strength criterion for unilateral materials}

As introduced above, a conventional strength criterion for unilateral material restricts trace and determinant of the stress tensor to be non-positive or non-negative in case of the no-tension or no-compression assumption, respectively. Within a two-dimensional framework, the relevant admissible sets of stress states may be effectively represented in the plane of the principal stres- ses $\sigma_{I}$ and $\sigma_{I I}$, see Figure 1. According to Eqns. (5), feasible points for a no-tension material are those of the third quadrant (compression-compression), while a no-compression medium finds admissible stresses in the first one (tension-tension). Eqns. (5) is not well-suited for the numerical treatment within a topology optimization procedure, mainly because the strength criterion is non-smooth (it has a singularity for $\sigma_{I}=\sigma_{I I}=0$ ) and consists of two inequalities per point.

Alternatively, one may regard unilateral materials as media having an extreme non-symmetric behavior in tension and compression. This straightforwardly suggests the adoption of a suitable form of the DruckerPrager strength criterion to handle a relaxed form of the relevant unilateral assumption.

Let consider a material whose uniaxial strength in compression and tension are defined as $\sigma_{L c}$ and $\sigma_{L t}$, respectively, being $s=\sigma_{L c} / \sigma_{L t}$ the so-called uniaxial asymmetry ratio. According to Drucker and Prager (1952), the stress state described by the tensor $\sigma_{i j}$ belongs to the feasible domain for the material strength if the following inequality on the equivalent stress measure $\sigma^{e q}$ holds everywhere in $\Omega$ :

$$
\begin{aligned}
& \sigma^{e q}=\alpha \sqrt{3 J_{2 D}}+\beta J_{1} \leq 1, \\
& \text { with } \alpha=\frac{\sigma_{L c}+\sigma_{L t}}{2 \sigma_{L t} \sigma_{L c}} \text { and } \beta=\frac{\sigma_{L c}-\sigma_{L t}}{2 \sigma_{L t} \sigma_{L c}},
\end{aligned}
$$

being $J_{1}$ the first stress invariant of $\sigma_{i j}$ and $J_{2 D}$ the second invariant of its deviatoric part. Assuming plane stress conditions one has:

$J_{1}=\sigma_{11}+\sigma_{22}$,

$3 J_{2 D}=\sigma_{11}^{2}+\sigma_{22}^{2}-\sigma_{11} \sigma_{22}+3 \sigma_{12}^{2}$.

The Drucker-Prager stress criterion has already been used in the recent literature to cope with materials exhibiting an uniaxial asymmetry ratio belonging to the 


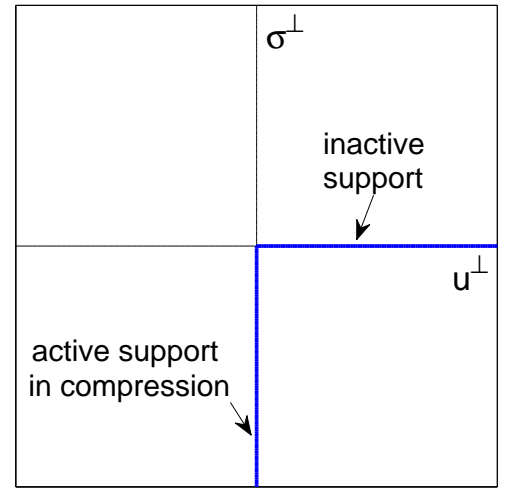

Fig. 2 Normal displacement $u^{\perp}$ vs. normal stress-flux $\sigma^{\perp}$ for the unilateral support of Eqns. (9).

range $1 / 5 \leq s \leq 5$, see Bruggi and Duysinx (2012) and Luo and Kang (2012). Reference is also made to Amstuz et al. (2012) that address the optimization of structures subject to Drucker-Prager stress constraints by means of an efficient topological derivative-based approach.

For the purpose of this contribution, it is worth discussing the representation of the Drucker-Prager criterion when a stronger asymmetry in the behavior tensioncompression is enforced. This has the aim of investigating whether unilateral materials may be coped with Eqn. (7), as asymptotic cases, for extremal values of the parameters $\sigma_{L t}$ and $\sigma_{L c}$.

Figure 1(a) represents the admissible stress state allowed by Eqn. (7) for a prescribed small value of the tensile strength $\sigma_{L t}$ along with the assumption $s=$ $\sigma_{L c} / \sigma_{L t}=100$. Figure $1(\mathrm{~b})$ plots the region of feasible stress for Eqn. (7) in case of a prescribed small value of the compressive strength $\sigma_{L c}$ along with $s=$ $\sigma_{L c} / \sigma_{L t}=1 / 100$. For $\sigma_{L t} \rightarrow 0$ a smooth approximation of the equations defining the third quadrant (i.e. the no-tension region) is achieved within the range of the principal stresses in Figure 1(a). Similarly, $\sigma_{L c} \rightarrow 0$ provides a smooth relaxation of the equations defining the first quadrant (i.e. the no-compression region) in the plot of Figure 1(b). This suggests that Eqn. (7) may be regarded as an effective alternative to classical enforcements of the type in Eqns. (5), to formulate a stress-based optimization procedure prescribing unilateral behavior of the material. The appropriate setting of the parameters entering Eqn. (7) will be further discussed in Section 4.

\subsection{Unilateral supports}

Let consider the case of unilateral supports prescribed along a portion of the boundary $\Gamma_{u}$, denoted as $\Gamma_{u c}$, with normal $\underline{n}$. The components of $\underline{n}$ in the orthogonal reference frame are denoted as $n_{i}$, meaning that $u^{\perp}=u_{i} n_{i}$ is the displacement along the normal, while $\sigma^{\perp}=\sigma_{i j} n_{i} n_{j}$ is the normal component of the stressflux across $\Gamma_{u c}$. Assuming a no-tension behavior of the support is equivalent to enforcing the following conditions along $\Gamma_{u c}$ :

$$
\begin{aligned}
& u_{i} n_{i}=0, \quad \sigma_{i j} n_{i} n_{j} \leq 0, \\
& \text { or } \\
& u_{i} n_{i} \neq 0, \quad \sigma_{i j} n_{i} n_{j}=0 .
\end{aligned}
$$

Prescriptions of Eqns. (9) are summarized in Figure 2, plotting sets of normal displacements $u^{\perp}$ and relevant normal stress-flux $\sigma^{\perp}$ that are admissible with respect to the assumed unilateral behavior of the support.

Within the framework of a topology optimization approach one may recall Eqn. (3) to re--write the conditions of Eqns. (9) into the following set of prescriptions along $\Gamma_{u c}$ :

$$
\begin{aligned}
& u_{i} n_{i}=0, \quad \rho^{p} \bar{\sigma}_{i j} n_{i} n_{j} \leq 0, \\
& \text { or } \\
& u_{i} n_{i} \neq 0, \quad \rho^{p} \bar{\sigma}_{i j} n_{i} n_{j}=0 .
\end{aligned}
$$

Alternatively, one may define a region of the twodimensional domain that is adjacent to the boundary $\Gamma_{u c}$, namely $\Omega_{u c}$, and therein enforce the constraints a for no-tension material, as introduced in Eqns. (6). This has the aim of avoiding the arising of tensile-stressed members that necessarily call for undesired tensile reactions along the boundary $\Gamma_{u c}$.

The above rationale suggests that the optimization of structures with unilateral supports may be straightforwardly accomplished within the weak form of Eqn. (2) through the prescription of the boundary condition $\left.\underline{u}\right|_{\Gamma_{u c}}=\underline{u}_{0}$ along with the appropriate enforcement of a set of Eqns. (7) over a limited region $\Omega_{u c}$.

If some material is distributed along $\Gamma_{u c}$ one has $\left.\rho\right|_{\Omega_{u c}}>0$, meaning that a compressive stress-flux $\sigma^{\perp}<$ 0 comes across the boundary and this is coupled with the enforced null normal component of the displacements, i.e. $u^{\perp}=0$. If $\Gamma_{u c}$ is adjacent to a no-material region one has $\left.\rho\right|_{\Omega_{u c}} \rightarrow 0$ and the support is simply inactive. In fact one finds $\sigma^{\perp}=0$ and the prescription of the boundary condition $u_{\perp}=0$ has no effect on the displacement field of the optimal design due to the interposed phase of soft material, see Eqn. (1). Following Section 2.1, one may re-formulate the dual problem involving no-compression supports by requiring tensileonly material in the vicinity of $\Gamma_{u c}$. 
As expected, a problem involving a no-tension material implicitly prescribes the unilateral behavior of supports along the whole $\Gamma_{u}$. In such a case Eqn. (10) are automatically fulfilled, since the optimization procedure distributes compression-only material for $\rho>0$. The same rationale applies to a no-compression material where the enforcement of Eqn. (7) in $\Omega$ automatically calls for tensile-only stress-flux across $\Gamma_{u}$.

It is finally remarked that the proposed implementation for unilateral supports is not conceived to solve general problems of unilateral contact. Reference is made to the literature mentioned in Section 1 for effective solutions of topology optimization problems involving the modeling of complex contact mechanics.

\section{Topology optimization for structures with unilateral behavior of material or supports}

The discretization of the weak problem derived in Eqn. (2) is accomplished in a standard way through the adoption of quadrangular finite elements with bi-linear displacement shape functions to approximate both fields $\underline{u}$ and $\underline{v}$. A piecewise constant discretization is adopted for the minimization unknown $\rho$, thus leading to the well-known discrete statement:

$\mathbf{K}(\mathbf{x}) \mathbf{U}=\sum_{e=1}^{N} x_{e}^{p} \mathbf{K}_{e}^{0} \mathbf{U}=\mathbf{F}$,

where $\mathbf{K}$ is the structural stiffness matrix, $\mathbf{U}$ is the generalized displacement vector and $\mathbf{F}$ is the vector addressing the external loads. Within the adopted discretization, the global matrix $\mathbf{K}$ may be conveniently assembled accounting for the $N$ element-wise contributions. They may be computed from $\mathbf{K}_{e}^{0}$, that is the (global level) element stiffness matrix for the virgin material, and $x_{e}$, that is the $e$-th component of the vector of the element densities x. After the solution of Eqn. (11) one may recover the structural compliance of Eqn. (4) as:

$\mathcal{C}=\sum_{e=1}^{N} x_{e}^{p} \mathbf{U}_{e}^{T} \mathbf{K}_{e}^{0} \mathbf{U}_{e}$

where element-wise contribution are highlighted in terms of $x_{e}, \mathbf{K}_{e}^{0}$ and $\mathbf{U}_{e}$, i.e. the element displacement vector.

The enforcement of the constraints introduced in Eqn. (7) calls for the postprocessing of the displacement field to recover the stress tensor all over the domain. Alternatively, one may resort to mixed discretizations that approximate stresses as direct variables of the elasticity problem, see e.g. Bruggi and Cinquini (2009). Following the displacement-based approach detailed in Duysinx and Sigmund (1998), one may switch to the Voigt notation and introduce the vector $\sigma_{e}=\left\{\begin{array}{lll}\sigma_{11} & \sigma_{22} & \sigma_{12}\end{array}\right\}^{T}$ that collects the components of the stress tensor in a relevant point of the $e$-th finite element, herein the centroid. Re-writing Eqn. (3), one has $\sigma_{e}=x_{e}^{p} \mathbf{T}_{e}^{0} \mathbf{U}_{e}$, where $\mathbf{T}_{e}^{0}$ is the so-called stress matrix of the element made of virgin material.

Appropriate strength criteria for SIMP should be defined on ad hoc stress measures to take into account that the evolving material behaves like a composite at the intermediate density range, see Rozvany et al. (1992). The work by Duysinx and Bendsøe (1998) introduces the so-called apparent "local" stress $\left\langle\sigma_{i j}\right\rangle=$ $\sigma_{i j} / x_{e}^{q}$, with $q>1$, that may straightforwardly replace $\sigma_{i j}$ to perform a physically consistent enforcement of stress constraints. A suitable discrete form of Eqns. (7) to be used in conjunction with SIMP therefore reads:

$$
\begin{aligned}
\left\langle\sigma_{e}^{e q}\right\rangle & =x_{e}^{(p-q)}\left(\alpha \sqrt{\mathbf{U}_{e}^{T} \mathbf{M}_{e}^{0} \mathbf{U}_{e}}+\beta \mathbf{H}_{e}^{0} \mathbf{U}_{e}\right)= \\
& =x_{e}^{(p-q)} \bar{\sigma}_{e}^{e q} \leq 1,
\end{aligned}
$$

where $\left\langle\sigma_{e}^{e q}\right\rangle$ is the equivalent Drucker-Prager "local" stress measure for the $e$-th finite element in $\Omega$. The above expression is written in terms of the matrix $\mathbf{H}_{e}^{0}$ and $\mathbf{M}_{e}^{0}$ that allows computing the linear form $J_{1, e}$ and the quadratic form $J_{2 D, e}$ from the element-wise displacements $\mathbf{U}_{e}$ as:

$$
J_{1, e}=x_{e}^{p} \mathbf{H}_{e}^{0} \mathbf{U}_{e}, \quad 3 J_{2 D, e}=x_{e}^{2 p} \mathbf{U}_{e}^{T} \mathbf{M}_{e}^{0} \mathbf{U}_{e} .
$$

\subsection{Problem formulation}

As above introduced, suitable sets of stress constraints may be coupled to conventional equations to enforce a prescribed unilateral behavior of material or supports when searching for optimal designs for maximum stiffness. Both kinds of problems may be framed within the following discrete setting:

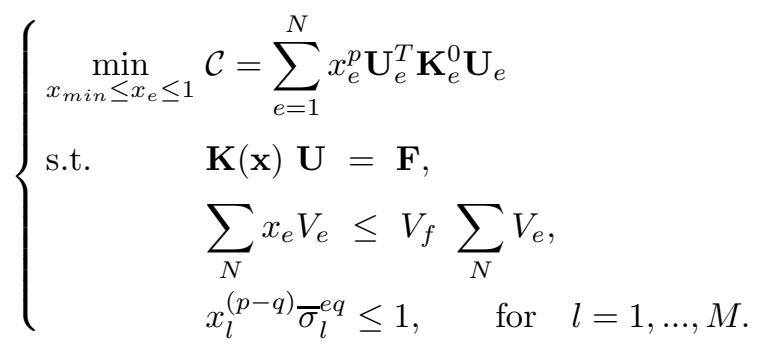

The objective function of the proposed formulation is the structural compliance $\mathcal{C}$, as computed in Eqn. (12). Eqn. (15.2) enforces the discrete equilibrium discussed in Section 2. Eqn. (15.3) is the constraint on the available amount of material, requiring the structural volume to be lower than a prescribed fraction $V_{f}$ of the full domain. The employed amount of material is computed multiplying the element density $x_{e}$ for the relevant volume $V_{e}$ over the $N$ elements in the mesh. 
Finally, Eqns. (15.4) refer to the set of $M$ local stress constraints enforced on the equivalent Drucker-Prager stress measure in the form of Eqn. (13).

A suitable setting of the strength parameters in $\bar{\sigma}_{l}^{e q}$ allows coping with no-tension or no-compression conditions, according to the discussion reported in Section 2.2. In case of unilateral material stress constraints are enforced all over the domain $\Omega$, i.e. $M=N$. When handling the optimization for unilateral supports one has $M<<N$ since constrained elements are those belonging to a limited area $\Omega_{u c}$ located in the vicinity of the boundary $\Gamma_{u c}$, see also Section 4 .

\subsection{Numerical issues}

The discrete form presented above has been tailored for the adoption of an element-wise density approximation along with the bi-linear interpolation of the displacement fields. The well-known checkerboard problem affects this standard discretization that also exhibits mesh dependence of the solution as a main consequence of a lack of well-posedness of the continuum SIMP-based problem, see in particular Sigmund and Petersson (1998) and Bendsøe and Sigmund (2003). To overcome both instabilities the density filter proposed in Bourdin (2001) and Bruns and Tortorelli (2001) is herein implemented, following the robust application in stress-based topology optimization provided by Le et al. (2010). Reference is also made to Sigmund (2007) and Guest et al. (2004) for a modification of the adopted density filter into a black-and-white projection filter.

The problem is solved via mathematical programming, adopting the Method of Moving Asymptotes by Svanberg (1987). The enforcement of a lower bound $x_{\min }>0$ is required on each density unknown $x_{e}$ to avoid singularity of the equilibrium equation. Section 4 will assume that $x_{\min }=10^{-3}$. To improve the performance of the algorithm, only a selected set of active stress constraints is passed to the minimizer, depending on the value of the 1.h.s in Eqns. (15.4). Following the strategy implemented in Bruggi and Duysinx (2012), a variable threshold increasing from 0,65 to 0,85 is adopted up to the tenth iteration, while it is constantly set to 0,85 thereafter. As shown in the numerical simulations, a robust control on the stress field is performed by the optimizer in the first steps of the procedure, thus steering the solution towards layouts of minimum energy that are feasible with respect to the relevant assumption of unilateral behavior. The number of selected constraints quickly decreases in the subsequent iterations and most of the optimization is performed as purely volume-constrained.

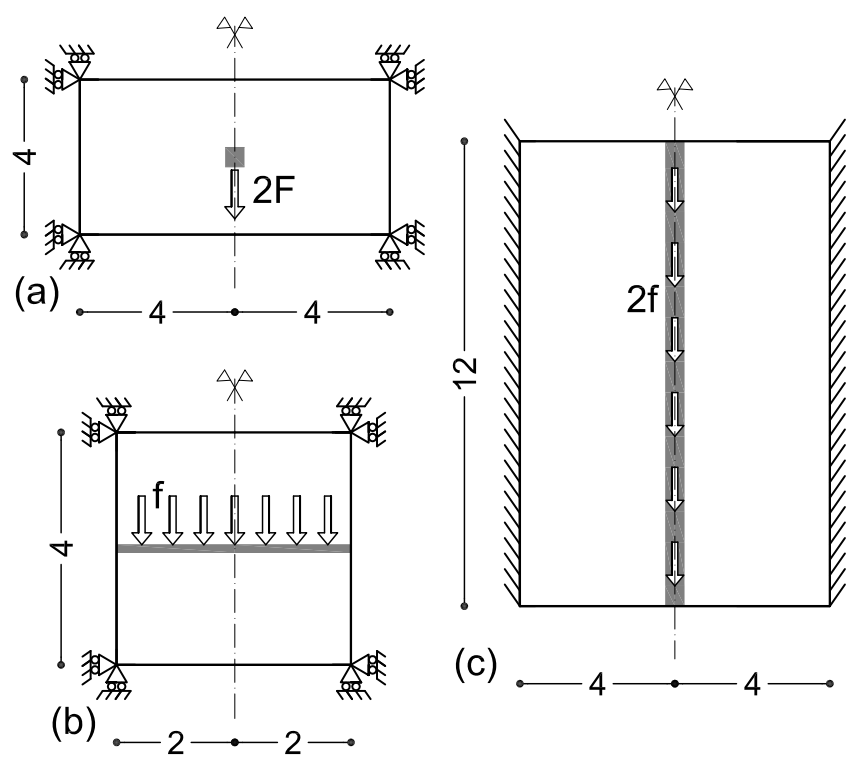

Fig. 3 Example 1-3. Geometry and boundary conditions for the numerical applications (dimension in $m$, unitary thickness, $F=$ $100 \mathrm{~N}, f=100 \mathrm{~N} / \mathrm{m})$.

Dealing with stress-based formulations one has also to take into account the well-known singularity problem, see in particular Rozvany (1996) and Cheng and Guo (1997). The standard assumption $p=q$ preserves full physical consistence of the SIMP-based strength model adopted in Eqn. (13), but also induces an undesired asymptotic behavior of the apparent "local" stress for vanishing material. The $q p$-approach is herein adopted to overcome numerical instabilities, meaning that an exponent $q<p$ is implemented to provide a strong relaxation in the low density region without introducing any remarkable bias at full density, see Bruggi (2008). This helps in preventing convergence towards undesired local minima made of grey material, while preserving a robust stress control over the full material zone. As expected, the tuning of the relaxing parameter $q$ remarkably affects the computational cost of the procedure, especially in the first demanding iterations, since it mainly governs the set of active constraints that are picked out by the selection strategy. Following Bruggi and Duysinx (2012), the simulations presented next assume $q=2.5$.

A final remark is given on the sensitivity computation of Eqns. (15.4). This should be performed via the adjoint method to take the best advantage of the selection strategy discussed above. Indeed, during the optimization, each active constraint requires the solution of one additional load case of the linear system governing the elastic equilibrium, see e.g. Haftka and Gürdal (1992). 


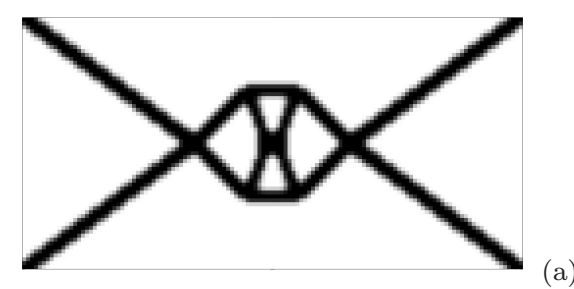

(a)

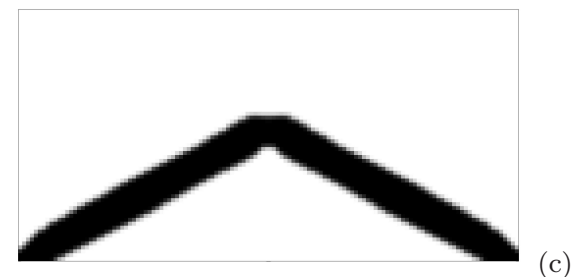

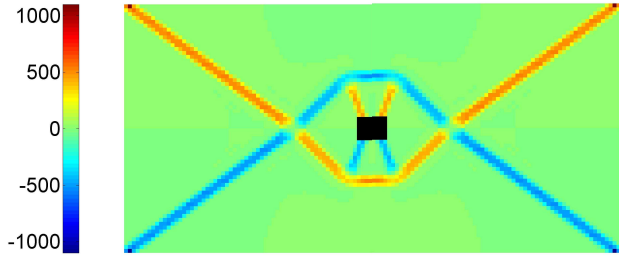

(b)

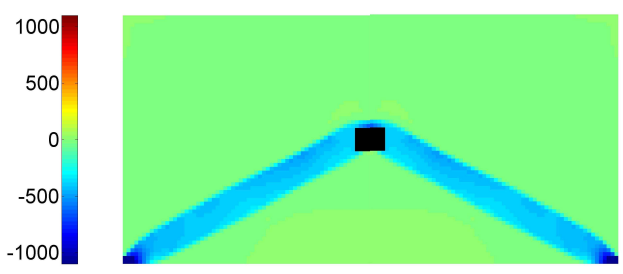

(d)

Fig. 4 Example 1. Optimal design and relevant map of the first stress invariant: symmetric material (a-b), no-tension material (c-d).

\section{Numerical simulations}

This section has the aim of assessing the formulation introduced in Section 3.1. The examples proposed in the sequel focus on results achieved in case of unilateral behavior of material, see Sections 4.1-4.3, or unilateral behavior of supports, see Sections 4.4-4.5.

Comparisons with problems solved for a symmetric constitutive behavior of materials and constraints are provided both in terms of optimal designs and convergence features of the implemented numerical procedures. Table 1 resumes the performance of the achieved layouts reporting their non-dimensional compliance $\mathcal{C} / \mathcal{C}_{0}$. Subscript 0 refers to the compliance computed on the full domain made of virgin material with symmetric behavior in tension and compression, assuming bilateral supports. Optimal designs are equipped with maps representing the element-wise values of the first stress invariant $J_{1, e}$, as computed in Eqn. (14). Since a uniaxial stress regime is expected in the achieved truss-like layouts, the invariant $J_{1, e}$ simply provides the relevant value of the axial (and principal) stress in each strut or tie. Concerning numerical issues, an optimization run is ended when each density unknown undergoes a variation, between two subsequent iterations, that is lower than a fixed tolerance, herein $10^{-3}$. The adopted discretization consists of regular meshes of square elements with unitary thickness, dealing with plane stress conditions. The number of finite elements $N$ is reported for each example in Table 1. A material with Young modulus $E=1 \mathrm{~N} / \mathrm{m}^{2}$ and Poisson ratio $\nu=0.3$ is considered. The allowed volume fraction is $V_{f}=0.15$.

An additional remark addresses the choice of suitable starting points for the unknowns of the topology optimization. This choice is implemented in the sequel according to different strategies, depending on the fea- tures of the adopted formulations. A classical strategy for volume-constrained compliance minimization assigns a starting density that is equal to the allowed volume fraction, i.e. $x_{e 0}=V_{f}, \forall e$, having the main aim of providing the minimizer with a feasible guess to begin with. Within the stress-based formulation of Eqn. (15) this guess is less efficient, due to the large amount of stress constraints that would be violated by a homogeneous distribution of intermediate densities. A better choice for such a setting consists in the adoption of unitary values for the starting guess of the whole set of variables, that is $x_{e 0}=1, \forall e$. This assumption does not satisfy the volume enforcement, but effectively decreases the overall amount of violated constraints in the first iterations, thus providing a noticeable speed up of the whole multi-constrained optimization.

\subsection{Example 1. No-tension material}

The example refers to geometry and boundary conditions defined in Figure 3(a), as similarly investigated by Cai et al. (2010). A rectangular lamina is constrained by four hinges located at the corners, while a vertical force acts upon the center of the domain. Due to symmetry, only half of the lamina is considered in the numerical simulations, adopting a mesh of about 4000 elements. Figure $4(\mathrm{a}-\mathrm{b})$ presents the optimal design achieved by a conventional formulation for minimum compliance, assuming that material has an equal behavior in tension and compression. The relevant map for the first stress invariant $J_{1, e}$ shows that the optimal layout fully exploits symmetry in the constitutive behavior, since tensile and compressive members share the load in equal parts. Indeed, the modulus of both maximum and minimum values of the axial stress is about $800 \mathrm{~N} / \mathrm{m}^{2}$ in the trusses. 


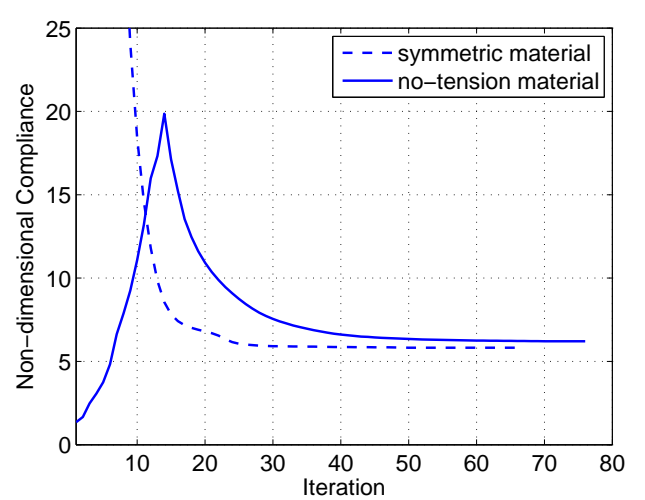

Fig. 5 Example 1. Compliance convergence curves for the optimization problems solved in Figure 4.

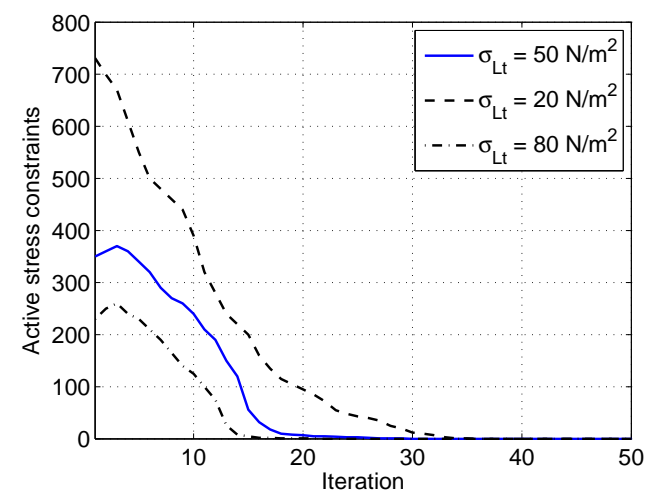

Fig. 6 Example 1. Topology optimization with no-tension material: number of active local stress constraints for different choices of the parameter $\sigma_{L t}$.

To cope with the optimization involving a no-tension material the formulation in Eqn. (15) is implemented, with a suitable setup of the parameters governing the strength criterion of Eqn. (7). Figure 4(c-d) shows the optimal design achieved for a prescribed small value of the tensile strength $\sigma_{L t}=50 \mathrm{~N} / \mathrm{m}^{2}$ along with the assumption $s=\sigma_{L c} / \sigma_{L t}=100$, see Section 2.2. As found in the aforementioned literature, the optimal truss-like structure consists of two thick struts connecting the load to the lower ground supports.

Figure 5 presents convergence curves of the nondimensional compliance $\mathcal{C} / \mathcal{C}_{0}$ for both the optimization problems solved in Figure 4 . The diagrams show diversities in the first iterations due to the alternative assumptions on the starting guess that have been previously discussed. Looking at the records of the multiconstrained optimization it can be observed that compliance grows in the first 15 steps, while it smoothly finds convergence in the remaining part of the optimization. As found in Figure 6 a number of stress constraints is active in the same first iterations, while the procedure turns to a cheap volume-constrained optimization thereafter. Indeed, stress constraints govern the first steps of the procedure and steer the optimization towards the achievement of stiff designs that are feasible with respect to the prescribed no-tension assumption. Additionally, Figure 6 shows the number of active constraints that have been recorded for two variations of the parameter $\sigma_{L t}$. All the simulations achieve the same optimal design that has been presented in Figure $4(\mathrm{c}-\mathrm{d})$, but their computational cost is different since it strongly depends on the above history plots. A reasonably small value of $\sigma_{L t}$ is required to avoid the arising of tensile-stressed members in the final layout, namely a low percentage of the maximum stress expected in case of a symmetric material behavior. However, the adoption of a too small $\sigma_{L t}$ could call for an excessive amount of active enforcements, thus increasing the cost of the optimization for a wider range of iterations. This also could make the procedure very sensitive to undesired stress peaks related to the bi-dimensional modeling, e.g. concentrations arising in the vicinity of the load application points or other geometric singularities.

Figure 7 focuses on the numerical features of the implemented stress-based procedure, addressing the optimal design in case of no-tension material through different simulations for $\sigma_{L t}=50 \mathrm{~N} / \mathrm{m}^{2}$. The above results, which refer to a discretization with $N=4096$ and an initial guess $x_{e 0}=1$ for each finite element, are taken as a reference in the proposed comparison.

A first investigation adopts an alternative initial guess, i.e. $x_{e 0}=0.5 \forall e$, that is implemented over $N=$ 4096 finite elements. The initial domain consists of a whole region of intermediate density and the number of active constraints recorded at the beginning of the optimization is, as expected, dramatically high. At the first step active constraints are approximately 1800 (vs. nearly 400 for the suggested choice of $x_{e 0}=1$ ). This number decreases in the subsequent iterations and vanishes around five steps beyond than in the reference case, see Figure 7(b). In fact, Figure 7(a) shows that the non-dimensional compliance curve for $x_{e 0}=0.5$ finds convergence with some delay with respect to the choice $x_{e 0}=1$. The final layout and its compliance are exactly the same as the optimal design reported in Figure $4(\mathrm{c}-\mathrm{d})$, i.e. $\mathcal{C} / \mathcal{C}_{0}=6.20$, meaning that the starting guess only affects the computational cost of the procedure, at least in the considered example. The assumption $x_{e 0}=V_{f}$ would be heavy to be implemented, since a number of constraints that is of the same order of $N$ is expected. It must be remarked that stress-constrained optimization deals with highly non-convex problems. Different starting points may be therefore implemented in the proposed procedure with the aim of detecting convergence towards undesired local minima. 


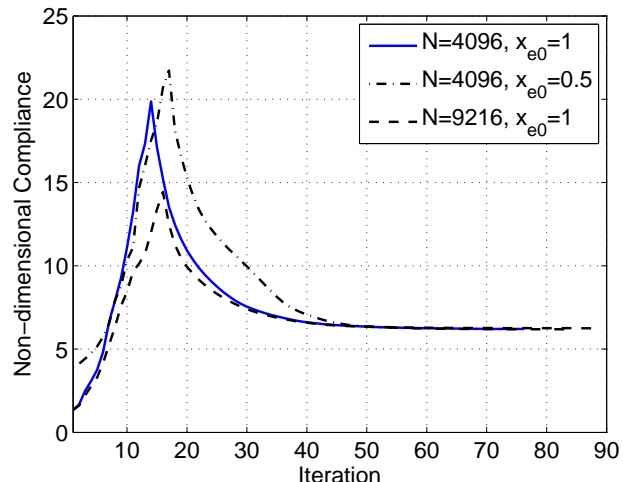

(a)

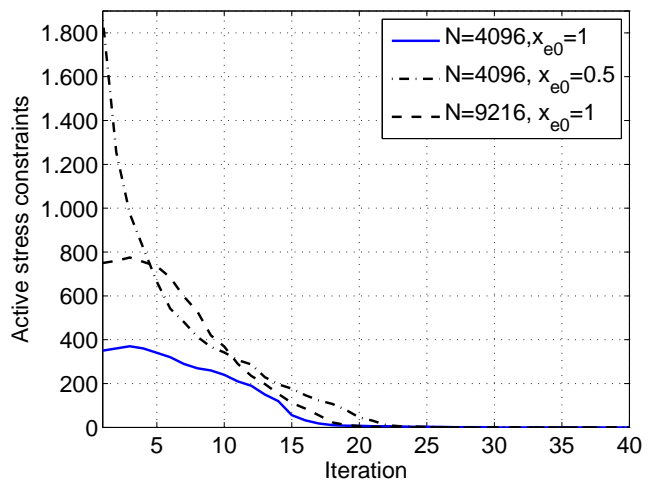

Fig. 7 Example 1. Topology optimization with no-tension material: compliance convergence curves (a) and number of active constraints (b) for different choices of the starting guess and the number of elements in the mesh.
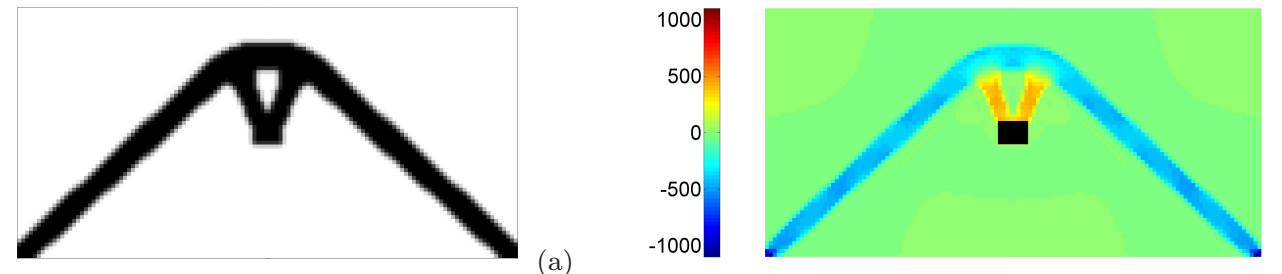

(b)

Fig. 8 Example 1. Optimal design and relevant map of the first stress invariant in case of symmetric material and ground constraints active only at the lower corners of the domain $(\mathrm{a}-\mathrm{b})$.

Figure 7 also investigates the effect of the adoption of a finer finite element discretization, for the suggested choice of $x_{e 0}=1$ as starting guess. Doubling the elements (and updating the filter radius in a consistent way) the number of active constraints grows accordingly, while the history reported in the convergence curve seems negligibly affected by mesh refinement. The optimal design is the same found in Figure $4(\mathrm{c}-\mathrm{d})$ and the non-dimensional compliance at convergence has a slight increase, i.e. $\mathcal{C} / \mathcal{C}_{0}=6.22$. This is mainly due to the improved accuracy of its discrete approximation as provided by the finer displacement-based mesh, see e.g. Bruggi and Verani (2011).

It is finally remarked that the proposed approach implements bi-linear finite elements. Each one adopts a constant density shape function and is coupled with a single evaluation of the stress constraint governing the unilateral behavior of the material, see Section 3. If more accuracy is required, one may easily and robustly switch to finer meshes as above investigated. Alternatively, quadratic elements may be employed. Their richer stress interpolation suggests to perform the constraints evaluation in more than one point for each element, meaning that a larger set of no-tension requirements would be introduced in the problem to control the element-wise constant density field. In such a case one could conveniently couple a finer discretization of the density field, thus providing a more consistent dis- tribution of unilateral material within each finite element.

As expected, the compression-only layout of Figure $4(\mathrm{c}-\mathrm{d})$ does not exploit the supports located at the upper corners of the lamina and one may wonder whether stress constraints simply prevent the optimizer from introducing tensile-stressed members beyond the load application point. A final investigation is therefore presented on the same problem, but removing the hinges originally located at the upper corners. Figure $8(\mathrm{a}-\mathrm{b})$ depicts the optimal result achieved by a pure volumeconstrained minimum compliance formulation that is not free from the arising of ties within the domain. Table 1 compares the non-dimensional compliance of the herein considered layouts, pointing out the effect of the no-tension assumption in terms of structural stiffness.

\subsection{Example 2. No-tension material}

The second example refers to an application of conceptual design for bridge structures, as originally investigated in Cai (2011). Geometry, loads and boundary conditions are those reported in Figure 3(b). Half of the domain is investigated adopting a mesh of about 8000 elements. Figure $9(\mathrm{a}-\mathrm{b})$ presents the conventional design achieved in case of material with equal behavior in tension and compression. The compressive arch-like 


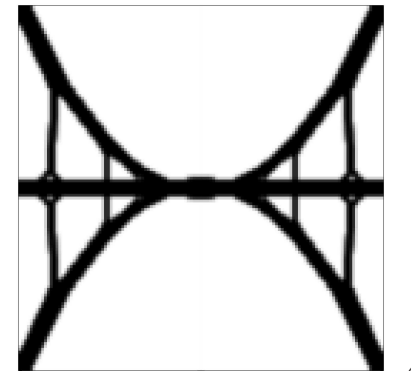

(a)

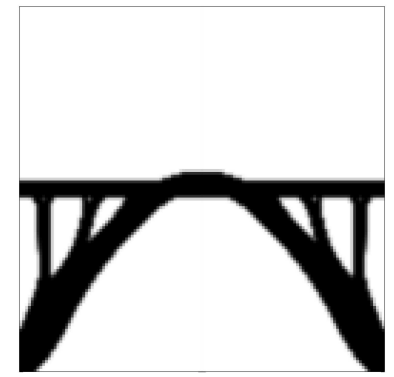

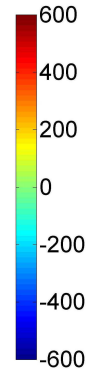

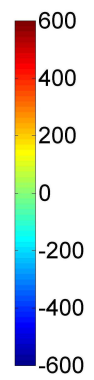

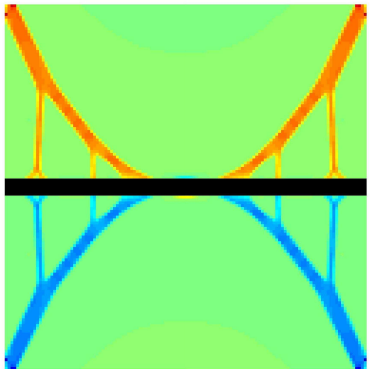

(b)

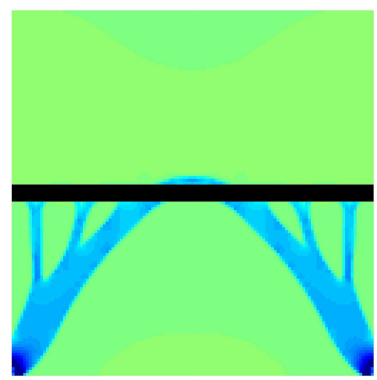

(d)

Fig. 9 Example 2. Optimal design and relevant map of the first stress invariant: symmetric material (a-b), no-tension material (c-d).

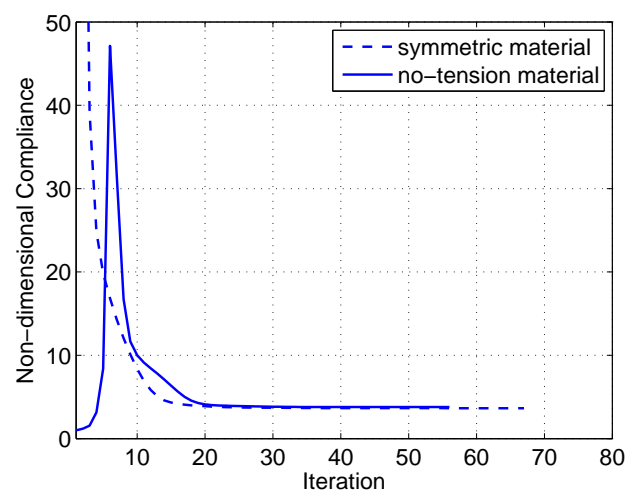

Fig. 10 Example 2. Compliance convergence curves for the optimization problems solved in Figure 9.

structure and the tensile cable are symmetrically connected to the bridge deck to transfer the distributed vertical loads towards the ground supports. The modulus of both maximum and minimum value of the axial stress is around $400 \mathrm{~N} / \mathrm{m}^{2}$.

To investigate the layout of the optimal structure in case of no-tension material, the stress-constrained formulation of Eqn. (15) is implemented, enforcing the same values of $\sigma_{L t}=50 \mathrm{~N} / \mathrm{m}^{2}$ and $s=\sigma_{L c} / \sigma_{L t}=100$ adopted before. The achieved inverted-catenary arch is presented in Figure 9(c-d), in full agreement with literature results. It is worth remarking that the compression-only design does not simply reduce to half of the symmetric layout found in case of equal behavior of material. This also calls for some re-modeling of the deck supports, see Chang et al. (2007).
Referring to convergence issues, Figure 10 compares curves reporting the history of non-dimensional compliance $\mathcal{C} / \mathcal{C}_{0}$ for both the optimization problems solved in Figure 9. As found in the previous example the multiconstrained formulation needs a very few iterations to address the optimizer towards the detailing of a stiff solution that respects the prescribed assumption of unilateral behavior of the structural components. The maximum size of the set of active constraints is around 350, all of them vanishing in less than 10 iterations.

\subsection{Example 3. No-compression material}

A last example to assess the procedure in case of unilateral material deals with the problem depicted in Figure 3(c). A uniform distribution of forces is prescribed along the vertical principal axis of the domain, while clamped edges are assigned at its left and right sides. The optimal solution for a conventional symmetric material is represented in Figure 11(a-b). The zone of prescribed fixed densities collects the vertical forces in the center of the domain, while couples of struts and ties bring the load to the external supports. Truss-like members are inclined of 45 degree with respect to the vertical axis, as expected dealing with shear-type actions. The whole design is homogeneously stretched and the average value of the principal stresses is around $250 \mathrm{~N} / \mathrm{m}^{2}$.

An optimal load path calling for tensile-only members is alternatively investigated resorting to the proposed stress-constrained formulation. All over the domain, the equivalent stress measure provided by the 

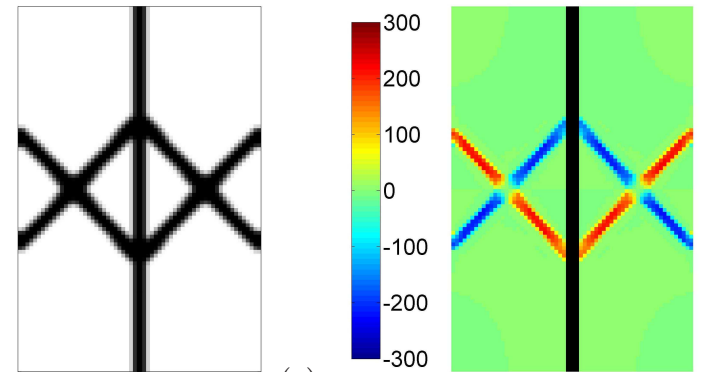

(a)
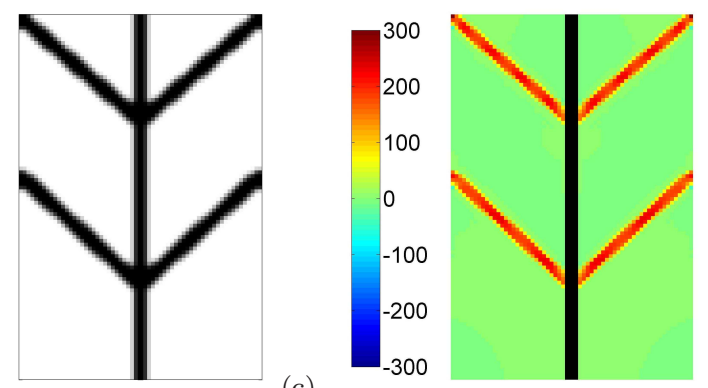

(c)

(d)

Fig. 11 Example 3. Optimal design and relevant map of the first stress invariant: symmetric material $(\mathrm{a}-\mathrm{b})$, no-compression material $(\mathrm{c}-\mathrm{d})$.

Drucker-Prager criteria is computed prescribing a small value of the compressive strength $\sigma_{L c}=50 \mathrm{~N} / \mathrm{m}^{2}$ along with $s=\sigma_{L c} / \sigma_{L t}=1 / 100$. Figure $11(\mathrm{c}-\mathrm{d})$ shows the achieved optimal design. To cope with the prescribed shear-type actions both layouts of Figure 11 share the need for inclined members. However, the removal of the struts from the pure volume-constrained solution is not enough to find an effective layout of minimum compliance. A better design consists of two tensile--only structures working in parallel. This allows for a reduction of the global strain energy as computed all over the domain, while providing full feasibility with the prescribed assumption on the material behavior.

This example has many similarities with the twobar truss problem that is a well-known benchmark to test stress-constrained formulations against the arising of the numerical instabilities briefly outlined in Section 3.2. Figure 11 shows that smooth convergence is found for the herein implemented multi-constrained optimization procedure.

\subsection{Example 4. Unilateral supports}

As detailed in Section 3.1, the formulation in Eqn. (15) may be adopted to cope with unilateral supports if stress constraints are enforced within regions adjacent to the relevant boundaries. To investigate the capabilities of the proposed approach, the cantilever presented in Figure 13(a) is firstly considered. The rectangular

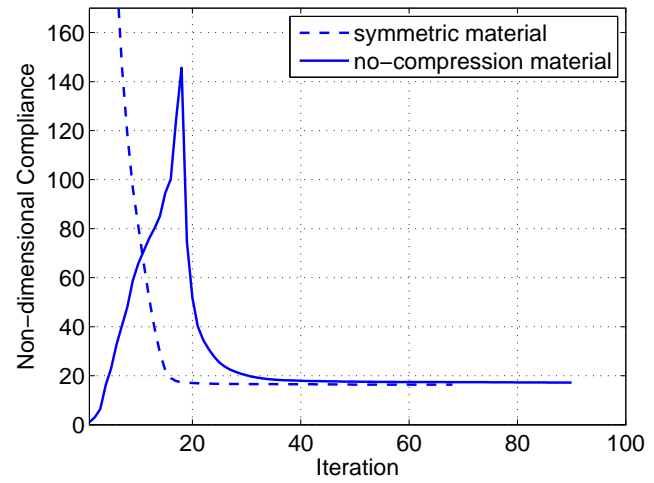

Fig. 12 Example 3. Compliance convergence curves for the optimization problems solved in Figure 11.
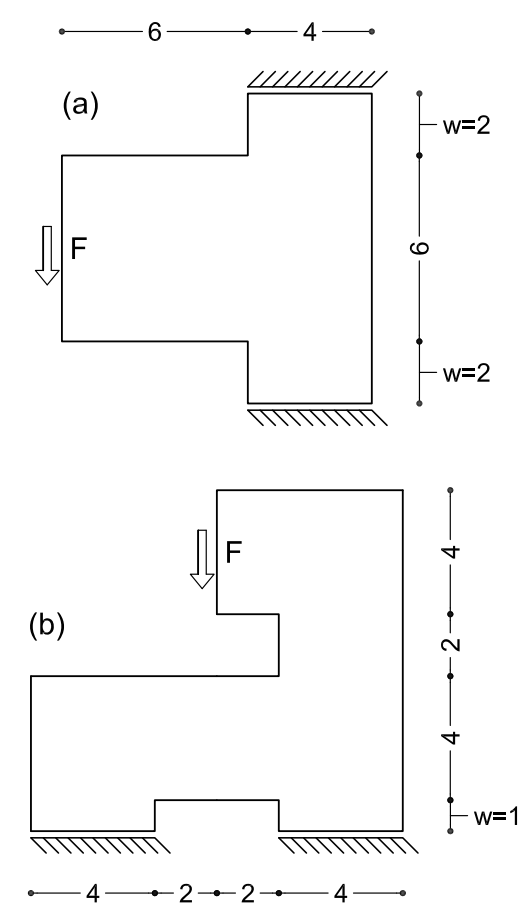

Fig. 13 Example 4-5. Geometry and boundary conditions for the numerical applications (dimension in $m$, unitary thickness, $F=100 \mathrm{~N})$.

lamina is thought as a corbel, needing optimization, that juts out of a wall. Two regions of thickness $w$ are included in the model to provide a discretization of the zones connecting the horizontal edges of the cantilever to a rigid stand. Horizontal displacements are unconstrained, while a vertical force is prescribed at the outer part of the corbel.

A preliminary investigation concerns the adoption of standard methods to compute the optimal design in case of bilateral supports, as presented in Figure 14(ab). The achieved truss-like structure splits the bending moment due to the external load in two anti-symmetric contributions that act upon the wall. Tensile reactions 

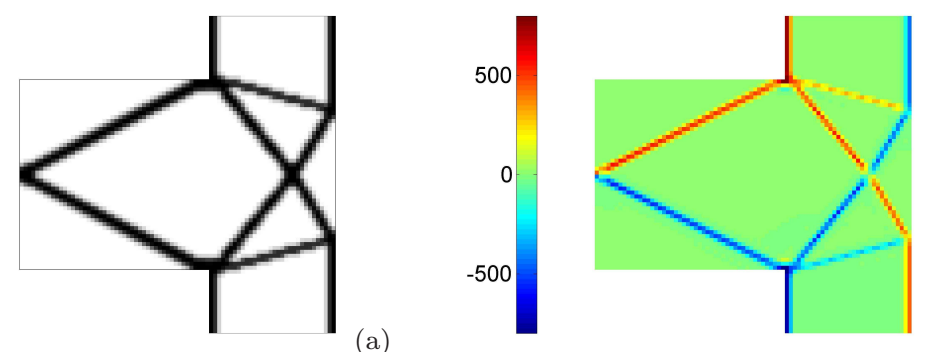

(a)
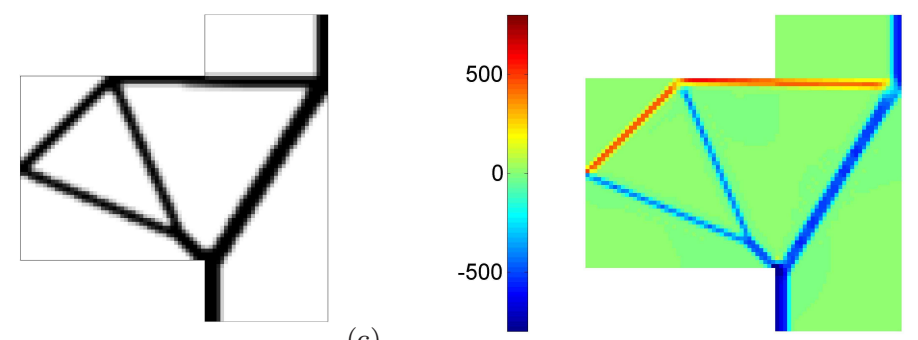

(b)

(c)

Fig. 14 Example 4. Optimal design and relevant map of the first stress invariant: bilateral supports (a-b), unilateral supports (c-d).

arise in this conventional solution along the constrained boundary, transferring stresses of about $600 \mathrm{~N} / \mathrm{m}^{2}$.

The same problem may be tackled by means of the formulation in Eqn. (15) to address the design in case of unilateral supports. Tensile reactions may be straightforwardly avoided by preventing the minimizer to deploy ties along the supports. A set of stress constraints is therefore enforced within the two regions of thickness $w$. The Drucker-Prager criteria is implemented for a small value of the tensile strength $\sigma_{L t}=50 \mathrm{~N} / \mathrm{m}^{2}$ along with $s=\sigma_{L c} / \sigma_{L t}=100$. Figure $14(\mathrm{c}-\mathrm{d})$ presents the optimal design found by the multi-constrained formulation. The bending moment due to the external load finds equilibrium thanks to the couple provided by the compression-only reactions arising along the two horizontal edges of the corbel. To maximize the stiffness, the arm of the couple is equal to the width of the wall. The achieved results are in good agreement with the work of Strömberg (2010) that originally investigated a contact problem between a cantilever of similar geometry and an outer elastic body. In both numerical experiments the truss-like structure found in the carried element is almost the same, mainly due to the enforcement of unilateral behavior of the constrained edges.

To provide an effective modeling of unilateral ground constraints the proposed approach calls for a non-zero thickness in the discretization of the support regions along $\Gamma_{u c}$. For $w>0$ a suitable domain $\Omega_{u c}$ is available to enforce stress-constraints that control the arising of unilateral reactions. If minimum density is distributed in $\Omega_{u c}$, a phase of soft material with $w>0$ makes the kinematic of the optimal design independent from

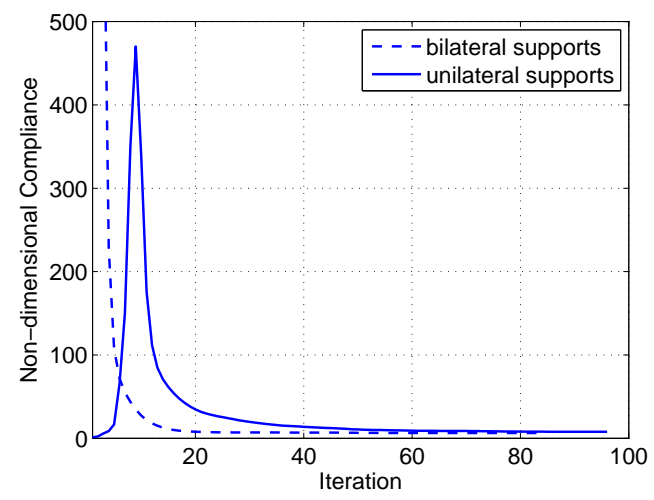

Fig. 15 Example 4. Compliance convergence curves for the optimization problems solved in Figure 14.

the displacement boundary conditions prescribed along $\Gamma_{u c}$, see Section 2.3.

Figure 15 presents convergence curves of the nondimensional compliance $\mathcal{C} / \mathcal{C}_{0}$ for both the optimization problems solved in Figure 14, while Figure 16 shows the history of the number of active constraints for the formulation in Eqn. (15). The same comments reported in the previous examples apply to this discussion. A few stress constraints enforce the required unilateral behavior in the very first steps of the procedure, while most of the optimization is performed as a pure volumeconstrained minimization.

Table 1 points out the effect of the considered assumptions concerning the behavior of ground constraints in terms of structural stiffness of the achieved optimal layouts. A limited increase in compliance is reported in case of unilateral supports. 

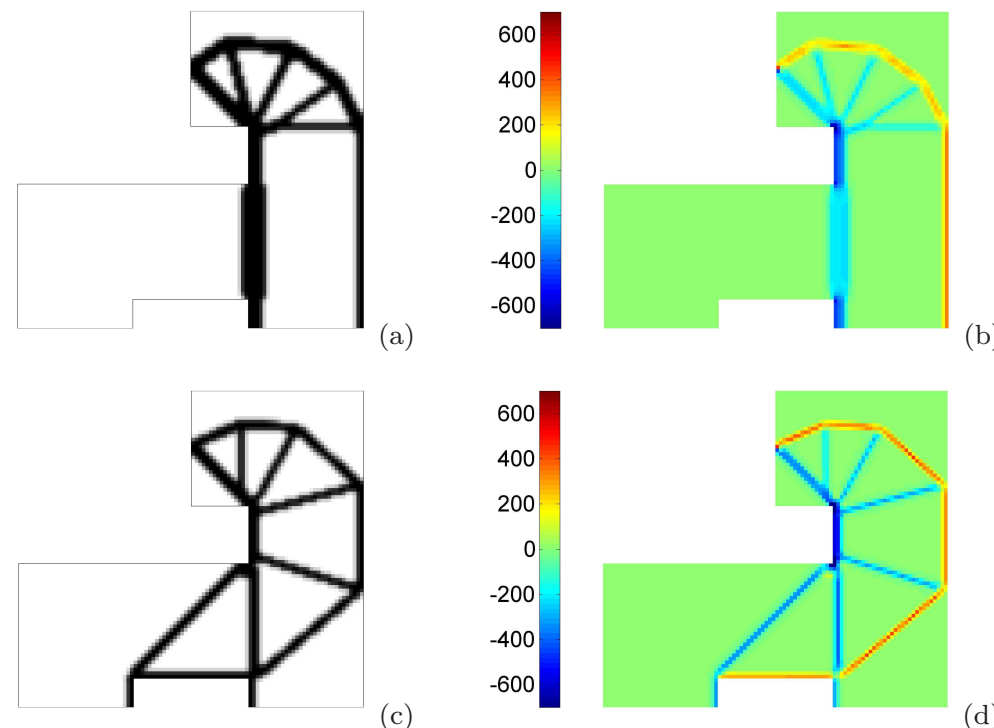

(b)

(c)

(d)

Fig. 17 Example 5. Optimal design and relevant map of the first stress invariant: bilateral supports (a-b), unilateral supports (c-d).

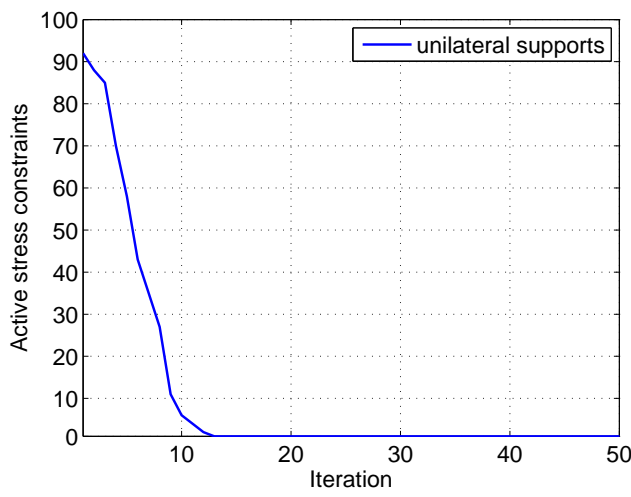

Fig. 16 Example 4. Topology optimization with unilateral supports: number of active local stress constraints for the optimization problem solved in Figure 14(c-d).

\subsection{Example 5. Unilateral supports}

The last example deals with the problem sketched in Figure 13(b). A first investigation concerns the achievement of the optimal design for minimum compliance in case of bilateral supports. The stiffest solution consists of a truss-like layout that transfers both compressive and tensile reactions to the ground, see Figure 17(ab). The foundation undergoes tensile stresses around $400 \mathrm{~N} / \mathrm{m}^{2}$.

To cope with the case of unilateral behavior of the constraints the formulation of Eqn. (15) is implemented. A set of stress enforcements is defined within the regions of thickness $w$ that lie adjacent to the ground supports. The strength parameters of the previous example are herein adopted, i.e. $\sigma_{L t}=50 \mathrm{~N} / \mathrm{m}^{2}$ and $s=\sigma_{L c} / \sigma_{L t}=100$.

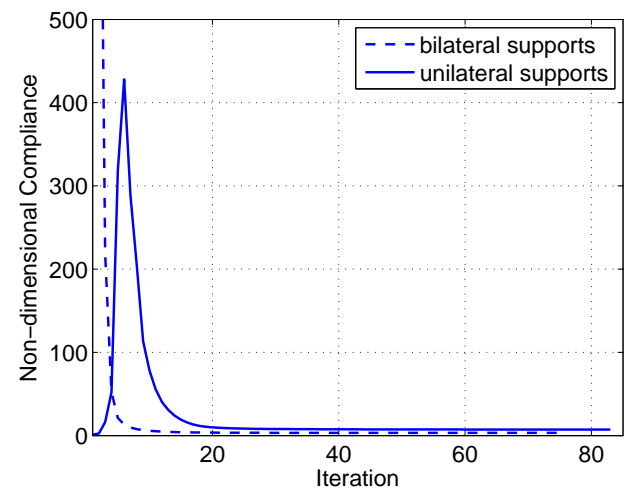

Fig. 18 Example 5. Compliance convergence curves for the optimization problems solved in Figure 17.

Figure $17(\mathrm{c}-\mathrm{d})$ shows that the achieved optimal design is much different with respect to the conventional solution provided for bilateral supports. To avoid tensile stresses at the foundation level no eccentricity is allowed between the resultant force of the reactions and the external vertical load. The price to pay is high in terms of compliance of the bearing structure, see Table 1 , while no convergence trouble is reported in the relevant history plot of Figure 18.

\section{Conclusions}

A stress-based approach has been presented to cope with the optimal design of truss-like elastic structures in case of unilateral behavior of material or supports.

The method consists in enriching the conventional volume-constrained minimization of compliance with a 


\begin{tabular}{c|c|c|c|c}
\hline Ex. & Fig. & Problem & $\mathrm{N}$ & $\mathcal{C} / \mathcal{C}_{0}$ \\
\hline 1 & $4(\mathrm{a}-\mathrm{b})$ & symmetric material & 4096 & 5.82 \\
1 & $4(\mathrm{c}-\mathrm{d})$ & no-tension material & 4096 & 6.20 \\
1 & $8(\mathrm{a}-\mathrm{b})$ & symmetric material & 4096 & 5.94 \\
\hline 2 & $9(\mathrm{a}-\mathrm{b})$ & symmetric material & 8192 & 3.64 \\
2 & $9(\mathrm{c}-\mathrm{d})$ & no-tension material & 8192 & 3.77 \\
\hline & $11(\mathrm{a}-\mathrm{b})$ & symmetric material & 3072 & 16.38 \\
3 & $11(\mathrm{c}-\mathrm{d})$ & no-compression material & 3072 & 17.11 \\
\hline & $14(\mathrm{a}-\mathrm{b})$ & bilateral supports & 4864 & 6.35 \\
4 & $14(\mathrm{c}-\mathrm{d})$ & unilateral supports & 4864 & 6.91 \\
\hline & $17(\mathrm{a}-\mathrm{b})$ & bilateral supports & 5632 & 3.24 \\
5 & $17(\mathrm{c}-\mathrm{d})$ & unilateral supports & 5632 & 5.49
\end{tabular}

Table 1 Examples 1-5. Comparison of the optimal designs in terms of non-dimensional compliance $\mathcal{C} / \mathcal{C}_{0}$, where subscript 0 refers to the full domain made of virgin material.

set of local stress constraints that have the aim of controlling the arising of members with tension-only or compression-only strength. In case of unilateral material, the optimal design is tackled enforcing constraints all over the domain. If unilateral supports are dealt with, the same setting is used to govern the stress regime within limited areas located in the vicinity of the ground constraints. No additional modification is required in the adopted simple formulation to handle the inherent non-linearity of the constitutive behavior of unilateral materials or ground supports.

A suitable form of the Drucker-Prager failure criterion has been investigated to provide a smooth approximation of the no-tension or no-compression conditions that control the admissible stress field in case of unilateral strength. The solution of the arising multiconstrained discrete problem has been tackled through mathematical programming, resorting to a selection strategy to increase computational efficiency.

Numerical simulations show that a limited set of constraints is needed in the first steps of the optimization to steer the algorithm towards the achievement of optimal designs that fulfill the prescriptions on unilateral behavior of material or supports. In both cases most of the procedure is performed as a pure volumeconstrained minimization of the strain energy, with benefits for the overall computational cost.

Benchmark examples assess the proposed method comparing results with solutions obtained in the recent literature by means of alternative numerical approaches. The achieved results point out that the assumption of unilateral behavior of material or supports plays a crucial role in the definition of minimum com- pliance designs to be exploited in many applications of structural engineering. Differences with respect to conventional solutions involving symmetric materials and bilateral constraints have been emphasized throughout the numerical section.

It is finally remarked that the proposed simplified framework is especially conceived to generate optimal truss-like designs whose mechanical behavior is mainly governed by the uniaxial stress state acting in each strut or tie. Optimal massive structures that are acted upon by complex bi-axial stress states should be carefully investigated resorting to more advanced material models.

\section{References}

Amstutz S, Novotny AA, de Souza Neto EA (2012) Topological derivative-based topology optimization of structures subject to Drucker-Prager stress constraints. Comp Meth Appl Mech Eng 236:123-136

Bendsøe M, Kikuchi N (1988) Generating optimal topologies in structural design using a homogeneization method. Comp Meth Appl Mech Eng 71:197-224

Bendsøe M, Sigmund O (1999) Material interpolation schemes in topology optimization. Arch Appl Mech 69:635-654

Bendsøe M, Sigmund O (2003) Topology optimization - Theory, methods and applications, Springer, EUA, New York

Bourdin B (2001) Filters in topology optimization. Int J Numer Methods Eng 50:2143-2158

Bruggi M (2008) On an alternative approach to stress constraints relaxation in topology optimization. Struct Multidiscip Optim 36:125-141

Bruggi M, Cinquini C (2009) An alternative truly-mixed formulation to solve pressure load problems in topology optimization. Comp Meth Appl Mech Eng 198:1500-1512

Bruggi M, Dusyinx P (2012) Topology optimization for minimum weight with compliance and stress constraints. Struct Multidisc Optim DOI: 10.1007/s00158-012-0759-7

Bruggi M, Verani M (2011) A fully adaptive topology optimization algorithm with goal-oriented error control. Comput Struct 89:1481-1493

Bruns TE, Tortorelli DA (2001) Topology optimization of nonlinear elastic structures and compliant mechanisms. Comp Meth Appl Mech Eng 190:3443-3459

Cai K (2011) A simple approach to find optimal topology of a continuum with tension-only or compression-only material. Struct Multidiscip Optim 43:827-835

Cai K, Shi J, Wang ZZ (2010) Tension/compression-only optimal stiffness design with displacement constraint. Proc. of the 2010 Int Conf on Intelligent Computation Technology and Automation, Changsha, China, 678-681

Chang CJ, Zheng B, Gea HC (2007) Topology optimization for tension/ compression only design. Proc. of the 7th WCSMO, COEX Seoul, Korea, 2488-2495

Cheng GD, Guo X (1997) $\varepsilon$-relaxed approach in topology optimization. Struct Optim 13: 258-266

Del Piero G (1989) Constitutive equation and compatibility of the external loads for linear elastic masonry-like materials. Meccanica 24: 150-162

Dewhurst P (2005) A general optimality criterion for combined strength and stiffness of dual-material-property structures. Int J Mech Sci 47:293-302 
Drucker DC, Prager W (1952) Soil mechanics and plastic analysis or limit design. Quart Appl Math 10:157-165

Duysinx P, Bendsøe MP (1998) Topology optimization of continuum structures with local stress constraints. Int J Numer Methods Eng 43: 1453-1478

Duysinx P, Sigmund O (1998) New developments in handling stress constraints in optimal material distribution. 7th Symposium on Multidisciplinary Analysis and Optimization AIAA-98-4906: 1501-1509

Eschenauer HA, Olhoff N (2001) Topology optimization of continuum structures: A review. Appl Mech Rev 54:331-389.

Fancello EA (2006) Topology optimization of minimum mass design considering local failure constraints and contact boundary conditions. Struct Multidiscip Optim 32:229240

Guan H, Steven GP, Xie YM (1999) Evolutionary structural optimisation incorporating tension and compression materials. Adv Struct Eng 2:273-288

Guest J, Prevost J, Belytschko T (2004) Achieving minimum length scale in topology optimization using nodal design variables and projection functions. Int $\mathrm{J}$ Numer Methods Eng 61(2):238-254

Haftka RT and Gürdal Z (1992) Elements of structural optimization, third revised and expanded edition, Dordrecht, Kluwer Academic publishers

Le C, Norato J, Bruns TE, Ha C and Tortorelli DA (2010) Stressbased Topology Optimization for Continua. Struct Multidiscip Optim 41:605-620

Liu S, Qiao H (2011) Topology optimization of continuum structures with different tensile and compressive properties in bridge layout design. Struct Multidiscip Optim 43:369380

Luo Y, Kang Z (2012) Topology optimization of continuum structures with Drucker-Prager yield stress constraints. Comp Struct 90-91:65-75

Mankame ND, Ananthasuresh GK (2004) Topology optimization for synthesis of contact-aided compliant mechanisms using regularized contact modeling. Comput Struct 82:1267-1290

Medri G (1982) A nonlinear elastic model for isotropic materials with different behavior in tension and compression. Trans ASME 26:26-28

Nowak M (2006) Structural optimization system based on trabecular bone surface adaptation. Struct Multidisc Optim 32:241249

Pàlfi P (2004) Locally orthotropic femur model. J Comput Appl Mech 5:103-115

Petersson J, Patriksson M (1997) Topology optimization of sheets in contact by a subgradient method. Int J Numer Methods Eng 40:1295-1321

Querin OM, Victoria M, Marti P (2010) Topology optimization of truss-like continua with different material properties in tension and compression. Struct Multidiscip Optim 42:25-32

Rozvany GIN (1996) Difficulties in truss topology optimization with stress, local buckling and system stability constraints. Struct Optim 11: 213-217

Rozvany GIN, Zhou M, Birker T (1992) Generalized shape optimization without homogenization. Struct Optim 4: 250-254

Rozvany GIN (2009) A critical review of established methods of structural topology optimization. Struct Multidiscip Optim $37: 217-237$

Sigmund O (2007) Morphology-based black and white filters for topology optimization. Struct Multidisc Optim 33(45):401424

Sigmund O, Petersson J (1998) Numerical instabilities in topology optimization: a survey on procedures dealing with checkerboards, mesh-dependencies and local minima. Struct Optim 16:68-75
Strömberg N (2010) Topology optimization of two linear elastic bodies in unilateral contact. Proc. of the $2^{\text {nd }}$ Int Conf on Engineering Optimization, Lisbon, Portugal

Strömberg N, Klarbring A (2010) Topology optimization of structures in unilateral contact. Struct Multidisc Optim 41:57-64

Svanberg K (1987) Method of moving asymptotes - A new method for structural optimization. Int J Numer Methods Eng 24:359-373

Xie YM, Steven GP (1993) A simple evolutionary procedure for structural optimization. Comput Struct 49:885-896

Zhou M, Rozvany GIN (1991) The COC algorithm, Part II : topological, geometrical and generalized shape optimization. Comp Meth Appl Mech Eng 89:309-336 